\title{
DICKE’S SUPERRADIANCE IN ASTROPHYSICS. II. THE OH 1612 MHz LINE
}

\author{
Fereshteh Rajabi ${ }^{1}$ and Martin Houde ${ }^{1,2}$ \\ ${ }^{1}$ Department of Physics and Astronomy, The University of Western Ontario, London, ON, N6A 3K7, Canada \\ 2 Division of Physics, Mathematics and Astronomy, California Institute of Technology, Pasadena, CA 91125, USA \\ Received 2016 January 10; revised 2016 April 6; accepted 2016 July 11; published 2016 August 31
}

\begin{abstract}
We apply the concept of superradiance that was introduced by Dicke in 1954 to the $\mathrm{OH}$ molecule $1612 \mathrm{MHz}$ spectral line, which is often used for the detection of masers in the circumstellar envelopes of evolved stars. Because the detection of $1612 \mathrm{MHz} \mathrm{OH}$ masers in the outer shells of envelopes of these stars implies the existence of a population inversion and a high level of velocity coherence, and that these are two necessary requirements for superradiance, we investigate whether superradiance can also happen in these regions. Superradiance is characterized by high-intensity, spatially compact, burst-like features taking place over timescales on the order of seconds to years, depending on the size and physical conditions present in the regions harboring such sources of radiation. Our analysis suggests that superradiance provides a valid explanation for previous observations of intensity flares detected in that spectral line for the U Orionis Mira star and the IRAS 18276-1431 preplanetary nebula.
\end{abstract}

Key words: ISM: molecules - molecular processes - radiation mechanisms: general

\section{INTRODUCTION}

The $\mathrm{OH}$ (hydroxyl) rotational transitions at nearly $18 \mathrm{~cm}$ were the first interstellar molecular lines detected in the radio range (Weinreb et al. 1963; Bertolotti 2015). The ground level of that molecule is split into two sub-levels known as $\Lambda$ doublets with $\pm \Lambda \hbar$ energies. Each component of the $\Lambda$ doublets is also split into two hyperfine levels labeled $F=1$ and $F=2$, as shown in Figure 1. The transitions that connect sub-levels with the same $F$-values are called the main lines, whereas the transitions between sub-levels of different $F$-values are called the satellite lines (Stahler \& Palla 2008). The four transitions, including the two main lines at 1665 and $1667 \mathrm{MHz}$ and the two satellite lines at 1612 and $1720 \mathrm{MHz}$, compose the group of $18 \mathrm{~cm}$ wavelength lines. In optically thin regions under conditions of local thermodynamic equilibrium (LTE), the expected intensity ratios are approximately 1:5:9:1 for the $1612,1665,1667$, and $1720 \mathrm{MHz}$ lines, respectively (Elitzur 1992). However, in several observations different line ratios were measured (McGee et al. 1965), and in some cases the intensity of a given line significantly exceeded that predicted using LTE (Weaver et al. 1965). The strong anomalous line intensities were explained by postulating maser action for the corresponding transitions.

Maser action occurs when LTE conditions are violated and velocity coherence is achieved between a group of populationinverted molecules. In the presence of a pumping mechanism that can maintain a higher population in the excited level, the corresponding transition can exhibit the exceptionally high intensity that is typical of maser sources (Elitzur 1992). The aforementioned study of Weaver et al. (1965) reported the first detection of an $\mathrm{OH}$ maser, which was to be followed by several other detections in different regions of the interstellar medium (ISM). A few years later Turner (1970) suggested classifying $\mathrm{OH}$ maser sources into two classes: Type I and Type II, depending on their brightest detected line. In Type I, the mainline transitions, especially the one at $1665 \mathrm{MHz}$, are dominant. The sources in this class are usually detected in star-forming sites near H II regions. Type II sources are further divided into Type IIa and Type IIb, in which the brightest line corresponds to one of the satellite lines. Type IIa $\mathrm{OH}$ maser sources, which are usually detected in supernova remnants, are brightest at $1720 \mathrm{MHz}$, while in Type IIb the $1612 \mathrm{MHz}$ line is dominant. These sources are often spatially associated with highly evolved stars that are undergoing rapid mass-loss and are enclosed in a circumstellar shell (Gray 2012). In addition, the first extragalactic $\mathrm{OH}$ maser was detected in 1982 by Baan et al. (1982) in Arp 220 (IC 4553), with a luminosity approximately $10^{8}$ times greater than that of typical Galactic $\mathrm{OH}$ masers. This led to the term "megamaser." Since then, several $\mathrm{OH}$ megamasers, and even gigamasers, have been detected (Darling \& Giovanelli 2002), usually in the nuclear region of luminous or ultraluminous infrared galaxies (Lockett \& Elitzur 2008).

The existence of $18 \mathrm{~cm} \mathrm{OH}$ masers confirms the possibility of inverting populations in the ground level $\Lambda$-doublets of this molecule in the ISM. Different pumping mechanisms are suggested for different types of Galactic and extragalactic masers. For instance, Type IIb maser sources near evolved stars are known to be pumped by the far-infrared radiation emitted from dust (Elitzur 1992; Gray et al. 2005; He 2005; see Section 2 for more details), while it is suggested that the pumping of $\mathrm{OH}$ Type I main-line masers is controlled by collisions with $\mathrm{H}_{2}$ molecules in star-forming regions (Kylafis \& Norman 1990). Maser action also requires line of sight velocity coherence, which can lead to an abnormally narrow line width through amplification along the radiation path. For Galactic $\mathrm{OH}$ $18 \mathrm{~cm}$ lines the typical line width is $\leqslant 1 \mathrm{~km} \mathrm{~s}^{-1}$ (McBride et al. 2013).

Population inversion and velocity coherence are also required for the superradiance cooperative radiation process. In 1954, R. H. Dicke pointed out that a sample consisting of $N$ excited atoms/molecules interacting with a common radiation field cannot always be considered as a collection of independent radiators (Dicke 1954). He showed that, under ideal conditions and after a time delay, the sample of atoms/ molecules can radiate its stored energy at an enhanced rate $N \Gamma$; $N$ times faster than the spontaneous emission rate $\Gamma$ of a single atom $/$ molecule. As a result, the intensity of the output radiation 


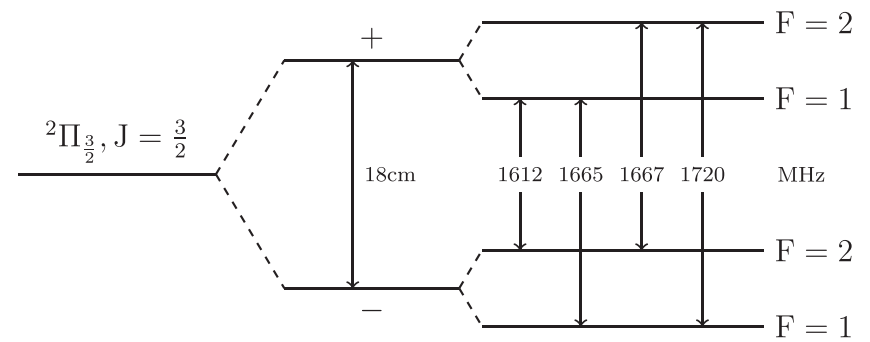

Figure 1. Schematic diagram of the ground rotational state of the $\mathrm{OH}$ molecule The ground rotational level labeled by ${ }^{2} \Pi_{3 / 2}, J=3 / 2$ splits into $\Lambda$-doublet sub-levels shown as \pm states. This splitting is due to the interaction between the rotational and electronic angular momenta of the molecule. Each $\Lambda$-doublet sub-level further splits into two hyperfine levels as a result of the interaction between the electron and nuclear spins of the hydrogen atom. The four possible maser transitions are shown with their corresponding frequencies in $\mathrm{MHz}$. Note that the hyperfine splitting is not to scale.

$I_{\mathrm{SR}}$ scales as the square of the number of inverted atoms/ molecules $N$, unlike the linear dependency of the radiation intensity from the corresponding non-coherent system $I_{\text {nc }}$ (Rajabi \& Houde 2016; hereafter Paper I).

In the ISM, it is usually assumed that atoms/molecules interact with the radiation field independently and the intensity of the radiation becomes a linear function of the atomic/ molecular density (if the line is optically thin). But in the case of the $\mathrm{OH} 18 \mathrm{~cm}$ line, for which the detection of several maser sources verifies the possibility of achieving population inversion and velocity coherence in some regions, this assumption may fail and it becomes important to examine the possibility of superradiance and coherent interactions. Accordingly, in this paper we follow up on the superradiance analysis performed in Paper I for the $\mathrm{HI} 21 \mathrm{~cm}$ (magnetic dipole) transition with a similar study for the $\mathrm{OH} 18 \mathrm{~cm}$ (electric dipole) line. In order to do so, we first discuss the necessary conditions for superradiance in Section 2, and narrow down our focus to the $1612 \mathrm{MHz}$ line interacting with $\mathrm{OH}$ molecules in the outer regions of the circumstellar envelope (CSE) of highly evolved stars. In Section 3, we investigate the likelihood that these conditions can be met in these regions using the Heisenberg approach, with a method of analysis that is an electric dipolar version of the magnetic dipole study found in Paper I and is similar to earlier analyses found in the physics literature (Gross \& Haroche 1982; Benedict et al. 1996). In Section 4 we discuss our numerical results on the characteristics of a potential $\mathrm{OH} 1612 \mathrm{MHz}$ coherent system, with an application to previous observations performed on the $U$ Orionis Mira star (Jewell et al. 1981) and the IRAS 18276-1431 preplanetary nebula (Wolak et al. 2014). We end with a short conclusion in Section 5.

\section{REQUIREMENTS FOR SUPERRADIANCE}

In this section we provide a brief summary of the requirements needed for superradiance, but a more detailed discussion will be found in Section 2 of Paper I. Our discussion applies equally well to atoms or molecules, however, we will focus on molecular superradiance since $\mathrm{OH}$ is the subject of our analysis.

When a group of $N$ excited molecules is placed within a volume $V$ much smaller than $\lambda^{3}$, the cube of the wavelength of the radiation $\lambda$ interacting with the molecules, the radiation by one molecule is seen to be in phase by the other molecules. As a result, the radiation from the different molecules interferes constructively and a strong directional pulse emerges from the sample (Dicke 1954). This process can also be described from the molecular point of view. In a small sample (i.e., defined for $V \ll \lambda^{3}$ ) the intermolecular distance $r$ is much smaller than $\lambda$, and for such small intermolecular distances, the interaction between the molecular dipoles and the radiation field is symmetrical throughout the sample. As a result, after a delay time $t_{\mathrm{D}}$ the molecular dipoles lock to a common phase and act like a single macroscopic dipole radiating a superradiant intensity $I_{\mathrm{SR}}=N I_{\mathrm{nc}}$, where $I_{\mathrm{nc}}$ is the intensity of a fully noncoherent system (Dicke 1954; Gross \& Haroche 1982; Benedict et al. 1996).

The phenomenon can be extended to a large sample, defined as $N$ molecules distributed over a volume $V \gg \lambda^{3}$, with intermolecular spacings potentially larger than $\lambda$. In a large sample, the phase of the radiation varies from place to place as a result of propagation. This will lead to a non-uniform spatial evolution of the molecules, and can result in a weaker coherent behavior compared to that of a small sample. However, the higher number of molecules partaking in coherent interactions in a large sample can make up for this, resulting in an intense output superradiant pulse with $I_{\mathrm{SR}}=N f I_{\mathrm{nc}}$, where the $N f$ factor determines the enhancement of the radiation intensity in comparison to $I_{\mathrm{nc}}$ and the efficiency of the common phaselocking process is reflected in $f(<1)$ alone. Unlike for a small sample, the phase-matching condition in a large sample can only be met in some particular directions, and the fact that the phase-locking factor $f$ is always smaller than unity implies a weakened superradiance. It is found that after a delay time $\tau_{\mathrm{D}}$, a first burst of superradiance emerges from the sample, followed by a number of weaker bursts, the so-called ringing effect (see Section 3 below, and Sections 3 and 4 in Paper I). The ringing effect is associated with the re-absorption and re-emission of radiation through the end-fire (i.e., the observer-facing crosssection of the superradiant sample) of a large sample interacting with the incoming radiation originating from other parts farther away in the sample.

Any mechanism that non-coherently reduces the excited level population (e.g., collisional relaxation) or disturbs the phase-locking process can diminish and even terminate superradiance. It is therefore necessary that the timescale of dephasing/relaxation effects be longer than $\tau_{\mathrm{D}}$ in a large sample (or $t_{\mathrm{D}}$ in a small sample) to allow for the development of any coherent behavior. This also explains why velocity coherence is an essential condition for superradiance. In the absence of velocity coherence, random thermal motions in a gas result in Doppler line broadening that corresponds to a very short dephasing timescale (i.e., $T_{\text {therm }} \ll \tau_{\text {D }}$ ), and renders this phenomenon as the most likely cause of dephasing in a sample. For this reason, it is not expected that superradiance could arise in a thermally relaxed gas. Our study of superradiance is better suited to regions of the ISM where thermal equilibrium has not been reached (e.g., shocks; see Paper I). However, this does not imply that Doppler broadening is not present in a velocitycoherent region, but it is expected to be less constraining than in a thermally relaxed environment.

For any region in the ISM, inelastic collisions with ions, electrons, hydrogen atoms and molecules, or dust grains can further change the internal state of an $\mathrm{OH}$ molecule, and if the associated timescale is smaller than $\tau_{\mathrm{D}}$, coherent behavior and superradiance can be suppressed. Although they do not change the internal state of a molecule, elastic collisions can also 
interrupt the coherent phase-locking process and weaken superradiance. This is because during an elastic collision the spacings between energy levels for the colliding counterparts change as a result of short-range interaction forces. After a number of such collisions, each acting like a random perturbation, the molecule can lose coherence with the interacting radiation field (Wittke \& Dicke 1956). Elastic collisions are normally more frequent than inelastic collisions, and the mean time between elastic collisions usually sets the timescale of collisional dephasing/relaxation $T_{\mathrm{c}}$ and the corresponding condition $\tau_{\mathrm{D}}<T_{\mathrm{c}}$ is required to allow the build-up of coherent interactions.

The above discussion also implies that coherent interactions cannot be developed in a collisionally pumped $\mathrm{OH}$ sample since that would require that the pumping timescale $T_{\mathrm{P}}=T_{\mathrm{c}}<\tau_{\mathrm{D}}$, which contradicts the necessary condition $T_{\mathrm{c}}>\tau_{\mathrm{D}}$ for superradiance (see Paper I). Thus, for our present study of superradiance we will only focus on $\mathrm{OH}$ samples that are believed to be inverted through radiative processes. The studies of pumping mechanisms of $\mathrm{OH}$ masers show that the $1612 \mathrm{MHz}$ masers associated with evolved stars are pumped by far-infrared photons at 35 and $53 \mu \mathrm{m}$ (Litvak 1969; Jewell et al. 1979; Elitzur 1992; Gray 2012). These photons are emitted from dust shells formed by mass losses from the central star. The radiative pumping model for $1612 \mathrm{MHz}$ masers near evolved stars is corroborated by the observation of correlated variations in the intensities of the star and corresponding masers (Harvey et al. 1974; Jewell et al. 1979). While recent studies by He (2005) and Lockett \& Elitzur (2008) suggest that $\mathrm{OH}$ megamasers, which emit primarily at 1667 and $1665 \mathrm{MHz}$, are also pumped by far-infrared radiation (more precisely 53 $\mu \mathrm{m}$ radiation from dust), the determination of the exact pumping mechanism of main-line masers still requires more studies and collisional processes are not generally ruled out from the pumping scenarios (Gray 2012). Hence, in this paper we limit our investigation to the possibility of superradiance for the $1612 \mathrm{MHz}$ line interacting with $\mathrm{OH}$ molecules in the CSEs of late-type stars.

\subsection{OH Samples near Evolved Stars}

One of the final stages in the evolution of a low- to intermediate-mass star (i.e., stars with masses of about $1 M_{\odot}$ to $\left.8 M_{\odot}\right)$ is the asymptotic giant branch (AGB) phase. In that stage, the star, which is composed of an oxygen/carbon core enclosed within layers of hydrogen and helium, becomes variable and can produce shock waves. Shock waves initiate the mass-loss process from the photosphere of the star to cooler regions where the gas particles can clump into dust grains, which interact with the radiation from the star over a broad continuum. Through these interactions the radially outgoing photons transfer their momentum to dust grains, driving them outward. This also results in an outflow of the gas particles that are coupled to dust grains through collisions (Lamers \& Cassinelli 1999; Gray 2012). As outflowing waves move farther from the star, they become cooler and denser and form the CSE. The CSE of an evolved star can harbor masers of different types, depending on its composition. $\mathrm{OH}$ masers are usually found in the CSE of oxygen-rich (or M-type) stars. Examples of such stars are Mira variables, long-period M-type stars with periods of 100-500 days (Karttunen 2007), which are known source of $1612 \mathrm{MHz} \mathrm{OH}$ maser emission. OH-IR stars are another group of long-period variables (LPV) that were originally detected through their $1612 \mathrm{MHz} \mathrm{OH}$ maser emission and the infrared radiation emanating from their CSE. Miras are variable at both visible and infrared wavelengths, whereas the CSE of OH-IR stars absorbs starlight at visible wavelengths and re-emits it in the infrared. OH-IR stars are thought to lose mass at a rate of $10^{-8} M_{\odot} \mathrm{yr}^{-1}$ to $10^{-4}$ $M_{\odot} \mathrm{yr}^{-1}$, forming larger CSEs than Miras, which have a lower mass-loss rate. The CSEs of these evolved stars are theoretically divided into three zones (Gray 1999), of which the inner and outer zones are relevant to our study. In the outermost zone, where the CSE is optically thin, UV light from the ISM dissociates $\mathrm{H}_{2} \mathrm{O}$ molecules into $\mathrm{OH}$. In the inner zones, the radiation from the central star is absorbed by dust grains and is re-emitted in the infrared. It was initially suggested by Elitzur et al. (1976) that the 35 and $53 \mu \mathrm{m}$ infrared photons emitted from dust pump the $1612 \mathrm{MHz} \mathrm{OH}$ masers in the outer regions of the CSE of evolved stars. The pumping scheme proposed by Elitzur et al. (1976) was modified by Gray et al. (2005), and their detailed analysis of collisional and radiative couplings of $\mathrm{OH}$ molecules in the expanding CSE of OH-IR stars indicated that the strongest pumping route uses $53 \mu \mathrm{m}$ photons.

The CSE of evolved stars expands radially and reaches a constant terminal velocity $v_{\infty} \sim 10 \mathrm{~km} \mathrm{~s}^{-1}$ to $20 \mathrm{~km} \mathrm{~s}^{-1}$ in its outer regions (Gray 2012). At distances about $r \gtrsim 10^{16} \mathrm{~cm}$ from the central star, velocity coherence is achieved among $\mathrm{OH}$ molecules moving with the well-defined terminal velocity in the radial or tangential directions relative to the $\mathrm{OH}$ shell (Draine 2011). This velocity coherence can be maintained over the so-called Sobolev length, which is typically on the order of $10^{14}-10^{16} \mathrm{~cm}$ for LPV evolved stars (see Section 4.1 below). This therefore may allow the existence of relatively long $\mathrm{OH}$ samples, if inversion is achieved through infrared pumping from warm dust. In such samples coherent correlations may develop if the shortest non-coherent relaxation/dephasing timescale is larger than $\tau_{\mathrm{D}}$.

Although our analysis is better adapted to regions where thermal equilibrium has not been reached, we will approximate the timescale of relaxation/dephasing effects in the usual manner applicable to a thermally relaxed gas. We should, however, keep in mind that the timescales calculated that way are likely to provide overestimates and should be considered as worst case scenarios, as far as superradiance is concerned. One of the main relaxation/dephasing mechanisms in the CSE of evolved stars is collision. We will therefore determine collision timescales in $\mathrm{OH}$ samples given a temperature $T$ and number density $n_{\mathrm{H}_{2}}$ of hydrogen molecules, which are expected to be the main collisional partners of $\mathrm{OH}$ in molecular gas shells (Gray 2012). These parameters depend on the mass-loss rate of the central star in a CSE (Goldreich \& Scoville 1976). For instance, at a mass-loss rate of $1 \times 10^{-5} M_{\odot} \mathrm{yr}^{-1}$ the abundance of $\mathrm{OH}$ molecules is estimated to be approximately $10 \mathrm{~cm}^{-3}$, while $n_{\mathrm{H}_{2}} \sim 10^{6} \mathrm{~cm}^{-3}$ at $r \approx 10^{16} \mathrm{~cm}$ based on the study of different physical and chemical processes taking place in the CSEs of OH-IR stars (Gray et al. 2005). Allowing for potentially lower mass-loss rates, in what follows we will consider the $10^{4} \mathrm{~cm}^{-3} \leqslant n_{\mathrm{H}_{2}} \leqslant 10^{6} \mathrm{~cm}^{-3}$ range.

The timescale of $\mathrm{OH}-\mathrm{H}_{2}$ collisions can be determined with knowledge of the collisional cross-sections in these regions. The cross-sections for inelastic $\mathrm{OH}-\mathrm{H}_{2}$ collisions at different temperatures are given in Offer et al. (1994), and the related timescale is estimated to range from $10^{5}$ to $10^{7} \mathrm{~s}$ for 
$10^{6} \mathrm{~cm}^{-3} \geqslant n_{\mathrm{H}_{2}} \geqslant 10^{4} \mathrm{~cm}^{-3}$, respectively. For elastic $\mathrm{OH}-\mathrm{H}_{2}$ collisions the corresponding timescale is given by

$$
T_{\mathrm{c}}=\frac{1}{n_{\mathrm{H}_{2}} \sigma_{\mathrm{g}} \bar{v}},
$$

where $\sigma_{\mathrm{g}} \simeq 4 \times 10^{-16} \mathrm{~cm}^{2}$ is the geometrical cross-sectional area of a hydrogen molecule and $\bar{v}$ is the mean relative velocity of OH molecules (Souers 1986; Irwin 2007). For example, $\bar{v}$ is estimated to be $\sim 1 \mathrm{~km} \mathrm{~s}^{-1}$ at $T \sim 100 \mathrm{~K}$. Inserting these values into Equation (1) gives $10^{4} \mathrm{~s} \leqslant T_{\mathrm{c}} \leqslant 10^{6} \mathrm{~s}$ for $10^{6} \mathrm{~cm}^{-3} \geqslant n_{\mathrm{H}_{2}} \geqslant 10^{4} \mathrm{~cm}^{-3}$, respectively. As was already mentioned, elastic $\mathrm{OH}-\mathrm{H}_{2}$ collisions are found to be somewhat more frequent than their inelastic counterpart and thus set the timescale of collisional dephasing $T_{\mathrm{c}}$.

Another important process that affects the population of $\mathrm{OH}$ energy levels in the CSE of evolved stars is the infrared radiative coupling of rotational levels. As was mentioned earlier, the warm dust in the CSE absorbs the radiation from the central star and re-emits it at mid- and far-infrared wavelengths. The different infrared couplings of the $\mathrm{OH}$ rotational levels have large transition dipoles, leading to fast excitation/ relaxation rates. It should be noted that these transition rates also depend on the opacity at the corresponding infrared wavelengths, which vary over the different zones of the CSE. Among the different infrared coupling routes, some pump the $1612 \mathrm{MHz}$ line while others deplete the population inversion and have a relaxation effect. The numerical studies conducted by Gray et al. (2005) show that these infrared couplings are responsible for the inverted $\mathrm{OH}$ zones, with a relaxation timescale for the $1612 \mathrm{MHz}$ line, including collisional and radiative couplings, on the order of $\sim 10^{4} \mathrm{~s}$ for a mass-loss rate of $10^{-5} M_{\odot} \mathrm{yr}^{-1}$. Allowing for variations with different massloss rates, we find that the radiative relaxation timescales are comparable to those expected from collisions in these regions. For the sake of our discussion we will assume, for simplicity, that the timescale of $\mathrm{OH}-\mathrm{H}_{2}$ collisions sets the upper limit for the characteristic timescales of superradiance in circumstellar $\mathrm{OH}$ samples, but we keep in mind that infrared radiative coupling may also be responsible for this. For the aforementioned range of $\mathrm{H}_{2}$ densities, when assuming thermal equilibrium the delay time $\tau_{\mathrm{D}}$ and the characteristic time of superradiance $T_{\mathrm{R}}$ should not exceed $10^{4} \mathrm{~s}$ to $10^{6} \mathrm{~s}$. Although these figures should be viewed as worst case scenarios (i.e., lower limits), they allow us to get a sense of the timescales involved. As we will see later, these timescales imply intensity variations that could last as long as several years.

\section{ANALYTICAL MODEL}

In this section we use the formalism developed in Gross \& Haroche (1982) to describe the behavior of a superradiant system. Since, as will be seen in Section 4, the realization of a superradiance small sample is unlikely to take place in the CSEs of evolved stars for the collisional timescales previously calculated (see Paper I), we focus our analysis on the case of a large sample.

Taking into account the dephasing/relaxation effects, the behavior of a superradiant system can be expressed by a set of so-called Maxwell-Bloch equations within the framework of the slowly varying envelope approximation (SVEA)

$$
\begin{aligned}
\frac{\partial \hat{\mathbb{N}}}{\partial \tau} & =\frac{i}{\hbar}\left(\hat{P}_{0}^{+} \hat{E}_{0}^{+}-\hat{E}_{0}^{-} \hat{P}_{0}^{-}\right)-\frac{\hat{\mathbb{N}}}{T_{1}} \\
\frac{\partial \hat{P}_{0}^{+}}{\partial \tau} & =\frac{2 i d^{2}}{\hbar} \hat{E}_{0}^{-} \hat{\mathbb{N}}-\frac{\hat{P}_{0}^{+}}{T_{2}} \\
\frac{\partial \hat{E}_{0}^{+}}{\partial z} & =\frac{i \omega}{2 \epsilon_{0} c} \hat{P}_{0}^{-},
\end{aligned}
$$

where $T_{1}$ is the (phenomenological) timescale of non-coherent population relaxation (e.g., through inelastic collisions) and similarly $T_{2}$ for phase relaxation (e.g., through elastic collisions). These equations were derived within the context of the Heisenberg representation. The quantities $\hat{P}_{0}^{ \pm}$and $\hat{E}_{0}^{ \pm}$are the envelopes for the polarization $\hat{\boldsymbol{P}}^{ \pm}$and the electric field $\hat{\boldsymbol{E}}^{ \pm}$ vectors, respectively, which are assumed to have the following form

$$
\begin{aligned}
\hat{\boldsymbol{P}}^{ \pm}(z, \tau) & =\hat{P}_{0}^{ \pm}(z, \tau) e^{ \pm i \omega \tau} \hat{\boldsymbol{\epsilon}}_{\mathrm{m}} \\
\hat{\boldsymbol{E}}^{ \pm}(z, \tau) & =\hat{E}_{0}^{ \pm}(z, \tau) \boldsymbol{e}^{\mp i \omega \tau} \hat{\boldsymbol{\epsilon}}_{\mathrm{m}},
\end{aligned}
$$

with $\hat{\epsilon}_{\mathrm{m}}$ as the unit vector indicating the orientation of the molecular electric dipole moment. The population inversion density is given by (twice) $\hat{\mathbb{N}}$, while $d$ and $\omega$ are, respectively, the transition dipole matrix element and the angular frequency of the radiation field at resonance with the molecular transition. Equations (2)-(4) are derived using a two-level system model and describe the evolution of the matter-field system in the retarded-time frame $\tau(=t-z / c$, where $c$ is the speed of light). In the ISM, an $\mathrm{OH}$ sample interacting with the radiation along the line of sight can be modeled by a cylindrical large sample along the $z$-axis. Although a one-dimensional field equation discards the loss of radiation due to diffraction or transverse effects, it is an approximation that reduces the number of variables and allows us to move the analysis forward while retaining the essential physics of the problem. Nonetheless, considerations of the sample's geometry, described by the Fresnel number $F=A /(\lambda L)$, with $A$ and $L$ as the cross-section and length of the sample, respectively, will also enter our analysis.

Under the assumption that the different dephasing timescales are similar (i.e., $T^{\prime} \equiv T_{1}=T_{2}$ ), the Maxwell-Bloch equations can be solved by effecting the following change of variables

$$
\begin{aligned}
\hat{\mathbb{N}} & =\frac{N}{2 V} \cos (\theta) e^{-\tau / T^{\prime}} \\
\hat{P}_{0}^{+} & =\frac{N d}{2 V} \sin (\theta) e^{-\tau / T^{\prime}},
\end{aligned}
$$

where $\theta$ is the so-called Bloch angle and $N$ is the number of inverted molecules at $\tau=0$ in the sample volume $V$. Inserting Equations (7) and (8) into the system of Equations (2)-(4) yields

$$
\begin{gathered}
\hat{E}_{0}^{+}=\frac{i \hbar}{2 d} \frac{\partial \theta}{\partial \tau} \\
\frac{d^{2} \theta}{d q^{2}}+\frac{1}{q} \frac{d \theta}{d q}=\sin (\theta),
\end{gathered}
$$




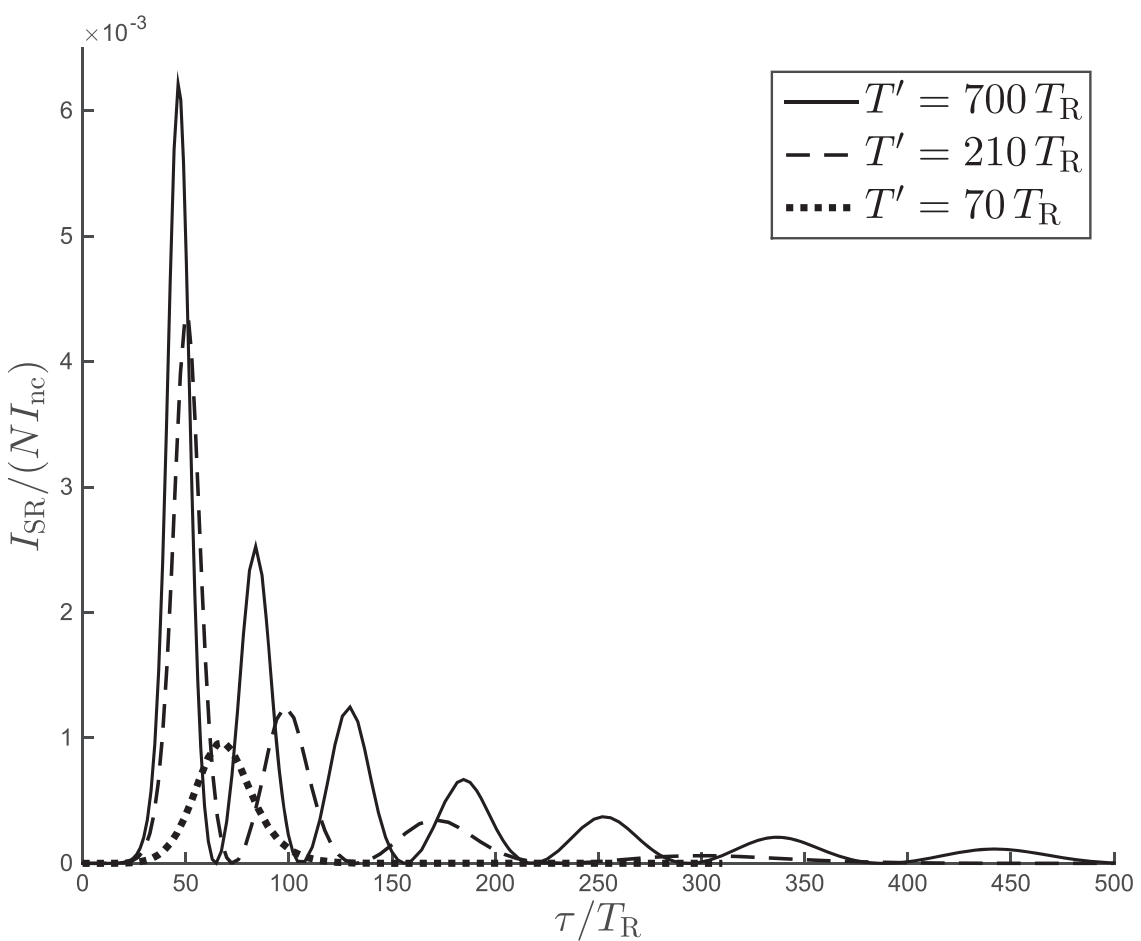

Figure 2. $\mathrm{OH}$ cylindrical large sample. The intensity axis, scaled to $N I_{\mathrm{nc}}$, is plotted vs. the retarded-time axis, normalized to the superradiance characteristic timescale $T_{\mathrm{R}}$. The length of the sample $L$ is set through Equation (12) for a given $T_{\mathrm{R}}$, and the Fresnel number is set to unity forcing the radius of the sample to $w=\sqrt{\lambda L / \pi}$. Dephasing effects are included for three different timescales $T^{\prime}=70 T_{\mathrm{R}}, 210 T_{\mathrm{R}}$, and $700 T_{\mathrm{R}}$.

with $q$

$$
q=2 \sqrt{\frac{z \tau^{\prime}}{L T_{\mathrm{R}}}},
$$

and $\tau^{\prime}=T^{\prime}\left(1-e^{-\tau / T^{\prime}}\right)$. The characteristic timescale of superradiance $T_{\mathrm{R}}$ is given by

$$
T_{\mathrm{R}}=\tau_{\mathrm{sp}} \frac{8 \pi}{3 n \lambda^{2} L},
$$

where $\tau_{\mathrm{sp}}$ is the spontaneous decay timescale of a single molecule and $n=N / V$ the density of inverted molecules in the sample (Gross \& Haroche 1982; Benedict et al. 1996). Equation (10) is the so-called Sine-Gordon equation, which can be solved numerically to find solutions for $\theta(q)$ at the endfire of the sample (i.e., at $z=L$ ). The solution for $\theta$ as a function $\tau$ can be used to evaluate $\hat{E}_{0}^{+}(z=L, \tau)$ from Equation (9), and then the intensity of radiation emerging from the sample with

$$
I_{\mathrm{SR}}=\frac{c \epsilon_{0}}{2}\left|\hat{E}_{0}^{+}\right|^{2},
$$

where $\epsilon_{0}$ is the permittivity of vacuum.

In Figure 2 we show three solutions for the radiation intensity from a cylindrical large sample of $\mathrm{OH}$ molecules interacting with the $1612 \mathrm{MHz}$ line, for the cases where $T^{\prime}=70 T_{\mathrm{R}}, 210 T_{\mathrm{R}}$, and $700 T_{\mathrm{R}}$. For these calculations we set the total number density of $\mathrm{OH}$ molecules to $n_{\mathrm{OH}}=10 \mathrm{~cm}^{-3}$, the level of inversion $\eta=0.01$, and $T_{\mathrm{R}}=7$ days. The length of the sample $L$ is set through Equation (12) for the given $T_{\mathrm{R}}$, and the Fresnel number is set to unity forcing the radius of the sample to $w=\sqrt{\lambda L / \pi}$. This value for the Fresnel number minimizes diffraction losses, which are not taken into account in our model (Gross \& Haroche 1982). In the figure the radiation intensity axis is scaled to $N I_{\mathrm{nc}}$ and the retarded-time axis is normalized to $T_{\mathrm{R}}$. We can express the non-coherent intensity $I_{\mathrm{nc}}$ as

$$
I_{\mathrm{nc}}=N \hbar \omega\left(\frac{1}{A \tau_{\mathrm{sp}}}\right)\left(\frac{\phi_{\mathrm{D}}}{4 \pi}\right),
$$

where $N \hbar \omega$ is the total energy initially stored in the $\mathrm{OH}$ sample, of which a fraction $\phi_{\mathrm{D}} / 4 \pi$ emerges from the end-fire of the sample through a cross-sectional area $A$ over the spontaneous decay timescale $\tau_{\mathrm{sp}}$. The superradiance beam solid-angle $\phi_{\mathrm{D}}$ is defined as

$$
\phi_{\mathrm{D}}=\frac{\lambda^{2}}{A}
$$

in the direction of the cylinder's symmetry axis where the phase-locking condition is fulfilled. Equation (14) can be rewritten as

$$
I_{\mathrm{nc}}=\frac{2}{3} \frac{\hbar \omega}{A T_{\mathrm{R}}},
$$

using Equations (12) and (15). Equation (16) makes it clear that the non-coherent intensity is roughly $N$ times smaller than the maximum superradiance intensity, since for the latter approximately $N$ inverted molecules decay to their ground level over the characteristic timescale of superradiance $T_{\mathrm{R}}$.

In a large sample, internal fluctuations (i.e., the thermal noise or spontaneous emission), as well as an external radiation, can trigger superradiance. For the calculations presented in Figure 2 we used internal fluctuations to set the initial Bloch angle at $\theta_{0}=2 / \sqrt{N}$ (Gross \& Haroche 1982). Once superradiance is 
initiated, dipoles in a large sample lock into a common phase and radiate coherently after the delay time (Benedict et al. 1996)

$$
\tau_{\mathrm{D}} \simeq \frac{T_{\mathrm{R}}}{4}\left|\ln \left(\frac{\theta_{0}}{2 \pi}\right)\right|^{2} .
$$

For the above $\mathrm{OH}$ sample we have $\theta_{0}=4.7 \times 10^{-5} \mathrm{rad}$, which results in $\tau_{\mathrm{D}} \simeq 35 T_{\mathrm{R}}$ using Equation (17), in good agreement with the time appearance of the first burst of radiation in Figure 2. As can be also seen in the figure, the total energy stored in the $\mathrm{OH}$ sample is released through consecutive bursts with a gradual drop in the peak intensities, with the number of bursts depending on the length of sample and the dephasing timescale $T^{\prime}$. More precisely, for longer samples and dephasing timescales (e.g., for $T^{\prime}=210 T_{\mathrm{R}}$ and $700 T_{\mathrm{R}}$ in the figure) the process of re-absorption/re-emission takes place more frequently at the end-fire of the sample, leading to a larger number of burst events. However, we should also note that the (nonlinear) Sine-Gordon equation is highly sensitive to initial conditions. It follows that the selected value for $\theta_{0}$ also has an impact on the appearance of the intensity curve (e.g., in the number of bursts present).

It should be also pointed out that the scaled peak intensities in Figure 2 indicate the phase-locking factor $0.001 \lesssim f \lesssim 0.01$, depending on the dephasing timescale. But the large number of inverted molecules $N$ in an $\mathrm{OH}$ large sample in a CSE will imply, even when multiplied by such a small value for $f$, a significant enhancement factor resulting in $I_{\mathrm{SR}} \gg I_{\mathrm{nc}}$.

\section{DISCUSSION}

The condition $\tau_{\mathrm{D}}<T^{\prime}$ further implies $T_{\mathrm{R}}<T^{\prime}$, since in a large sample composed of $N \gg 1$ molecules $\tau_{\mathrm{D}}$ is at least an order of magnitude larger than $T_{\mathrm{R}}$ (see Equation (17)). More precisely, the average delay time, i.e., for several realizations of a superradiance system with a different $\theta_{0}$, is given by (Gross \& Haroche 1982)

$$
\left\langle\tau_{\mathrm{D}}\right\rangle=T_{\mathrm{R}} \ln (N),
$$

which again indicates that $T_{R}<\left\langle\tau_{\mathrm{D}}\right\rangle$.

In a small sample, where there are a relatively small number of atoms, the two timescales $T_{\mathrm{R}}$ and $\left\langle\tau_{\mathrm{D}}\right\rangle$ are approximately of the same order of magnitude and the condition $\tau_{\mathrm{D}}<T^{\prime}$ can be interchanged with $T_{\mathrm{R}}<T^{\prime}$. For such cases the superradiance timescale can be calculated from (Dicke 1954)

$$
T_{\mathrm{R}}=\frac{\tau_{\mathrm{sp}}}{\eta n_{\mathrm{OH}} V},
$$

where, once again, $\eta$ is the population inversion factor, $n_{\mathrm{OH}}$ is the total molecular density, and $V$ is the volume of the sample. A small sample of $\mathrm{OH}$ molecules interacting with the $1612 \mathrm{MHz}$ line is characterized by $V<\lambda^{3} \sim 10^{3} \mathrm{~cm}^{-3}$. Applying this constraint on the volume and substituting $\tau_{\text {sp }} \sim 10^{11} \mathrm{~s}$ for the $1612 \mathrm{MHz}$ transition line transforms Equation (19) to

$$
T_{\mathrm{R}}>\frac{10^{8}}{\eta n_{\mathrm{OH}}} \mathrm{s},
$$

yielding $T_{\mathrm{R}}>10^{9} \mathrm{~s}$ for $n_{\mathrm{OH}} \sim 10 \mathrm{~cm}^{-3}$ (Gray et al. 2005) and $\eta \sim 0.01$, which are appropriate for masing regions in CSEs.
On the other hand, we previously calculated the timescale of $\mathrm{OH}-\mathrm{H}_{2}$ collisions $T_{\mathrm{c}}$ in circumstellar $\mathrm{OH}$ samples to be $\sim 10^{4} \mathrm{~s}$ to $10^{6} \mathrm{~s}$ for molecular hydrogen densities of $10^{6} \mathrm{~cm}^{-3}>n_{\mathrm{H}_{2}}>10^{4} \mathrm{~cm}^{-3}$ (see Section 2.1). Although our estimated range for this collision timescale is likely underestimated, it indicates that superradiance is unlikely to take place in corresponding $\mathrm{OH}$ small samples since $T_{\mathrm{c}}=T^{\prime}<T_{\mathrm{R}}$.

In a large sample $T_{\mathrm{R}}$ is set by the two sample parameters: length $L$ and density of inverted molecules $n$ (see Equation (12)). Although it may initially appear that in a large sample $T_{\mathrm{R}}$ can always be set to a value smaller than $T^{\prime}$ by adjusting $L$ or $n$, in the ISM these parameters are constrained by the physical characteristics of the region within which the population inversion is realized.

In the case of CSE OH samples, the length of an inverted region depends on the mass-loss rate of the central pulsating star, which changes as the star evolves. The computational modeling of CSEs of OH-IR stars by Gray et al. (2005) suggests that the radial extent of $\mathrm{OH}$ population-inverted zones shrinks as the mass-loss rate of the central star increases. More precisely, the mass-loss rate affects the optical depth of the infrared pump photons and subsequently the thickness of inverted $\mathrm{OH}$ zones. Hence, in higher mass-loss rates the envelope becomes more opaque to pump photons and the extent of the region accessible to the pump decreases. It is expected that population-inverted regions typically range from $10^{11} \mathrm{~cm}$ to $10^{14} \mathrm{~cm}$ in thickness (Gray et al. 2005).

Using Equation (12) we can use this range for the length $L$ of a cylindrical large sample, along with our previous values of $n=\eta n_{\mathrm{OH}}=0.1 \mathrm{~cm}^{-3}$ (i.e., 643 molecules within $\lambda^{3}$ ) and $\tau_{\text {sp }}=7.8 \times 10^{10} \mathrm{~s}$ to find $10^{-1} \mathrm{~s} \gtrsim T_{\mathrm{R}} \gtrsim 10^{-3} \mathrm{~s}$ for $10^{11} \mathrm{~cm}<L<10^{14} \mathrm{~cm}$. Using a Fresnel number of unity to minimize diffraction losses in our calculations, specifying a radius $w=\sqrt{\lambda L / \pi}$ ranging from $7.7 \times 10^{5} \mathrm{~cm}$ to $2.4 \times 10^{7} \mathrm{~cm}$, we find that $10 \mathrm{~s} \gtrsim\left\langle\tau_{\mathrm{D}}\right\rangle \gtrsim 10^{-2} \mathrm{~s}$ for the same range of cylindrical lengths. The timescales are evidently very short in comparison to our previous estimates of $10^{4} \mathrm{~s}<T_{\mathrm{c}}<10^{6} \mathrm{~s}$. It thus appears reasonable to expect that superradiance could take place in the CSEs of such evolved stars. Indeed, we find that $\left\langle\tau_{\mathrm{D}}\right\rangle \sim 10^{6} \mathrm{~s}$ for as small a value as $L=10^{5} \mathrm{~cm}$. Given these numbers, we now investigate potential observational evidence for superradiance in the $\mathrm{OH}$ $1612 \mathrm{MHz}$ line.

\subsection{The U Orionis Mira Star}

U Orionis is a M8 III-type OH Mira variable with a period of 372 days, which, in the past, has exhibited significant variations in the intensity of several $\mathrm{OH}$ maser emission lines. Jewell et al. (1981) reported the results of their monitoring program of the 1612,1665 , and $1667 \mathrm{MHz}$ masers observed in the CSE of U Ori, along with a compilation of similar data taken from several other sources (Pataki \& Kolena 1974; Reid et al. 1977; Cimerman 1979; Fix 1979) covering several years of observations (i.e., from 1974 to 1979). All three maser lines exhibited significant flaring events during that period. Although the 1665 and $1667 \mathrm{MHz}$ masers showed strong correlation in their intensity variations, the $1612 \mathrm{MHz}$ maser displayed a completely different behavior that was characterized by the authors as a "damped oscillator decline" spanning about an order of magnitude in intensity range over the period. These variations were also clearly uncorrelated with the star's light 


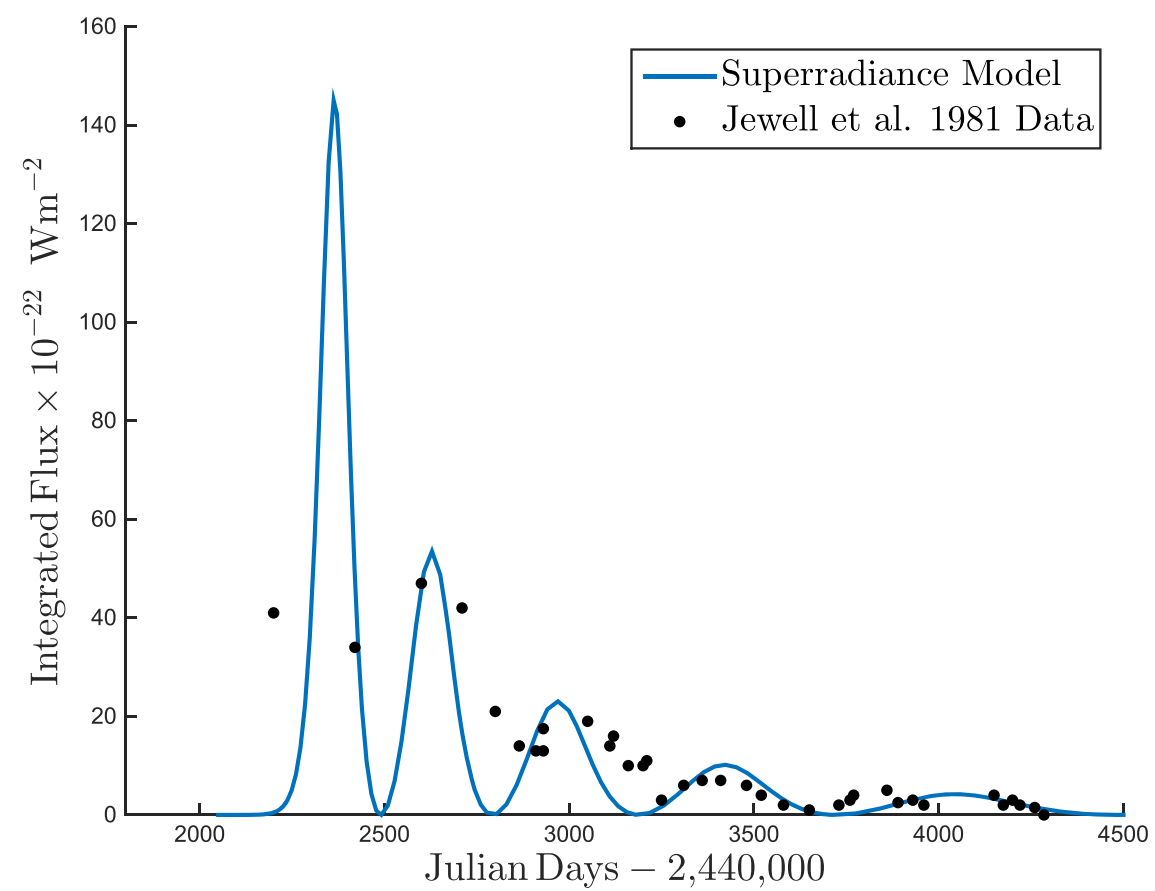

Figure 3. Superradiance intensity model (solid blue curve) with $T_{\mathrm{R}}=6.5$ days, $T^{\prime}=393 T_{\mathrm{R}}$, and $\theta_{0}=4.4 \times 10^{-5}$ rad superposed, on data from Jewell et al. (1981; black dots) that was obtained during the $1974-1979 \mathrm{OH} 1612 \mathrm{MHz}$ maser flaring episode of U Orionis.

curve (see Figure 1 of Jewell et al. 1981), and there has been no satisfactory explanation for this behavior that we are aware of so far.

Since the ringing in superradiance intensity displayed in the $T^{\prime}=210 T_{\mathrm{R}}$ and $700 T_{\mathrm{R}}$ curves shown in Figure 2 is also reminiscent of a "damped oscillator" behavior, the data of Jewell et al. (1981) provide us with a first opportunity to test our $\mathrm{OH} 1612 \mathrm{MHz}$ superradiance model. We accordingly show in Figure 3 the results of our attempt. In the figure, the black dots are taken from Figure 1 of Jewell et al. (1981), while the (blue) solid curve is calculated from our model discussed in Section 3. We have once again used the internal fluctuation condition to trigger superradiance (i.e., $\theta_{0}=2 / \sqrt{N}$ ), which resulted in $\theta_{0}=4.4 \times 10^{-5} \operatorname{rad}\left(\tau_{\mathrm{D}} \simeq 35 T_{\mathrm{R}}\right.$; see Equation (17)) for the chosen parameters $T_{\mathrm{R}}=6.5$ days and $T^{\prime}=393 T_{\mathrm{R}}$, while keeping $n=0.1 \mathrm{~cm}^{-3}$. Given the simplicity of our onedimensional superradiance model, we have not attempted to perform any formal fit to the data, but have merely adjusted the model's free parameters (i.e., $T_{\mathrm{R}}$ and $T^{\prime}$ ) to reproduce the main features found in the data. Our model was also "normalized" in intensity to that of the data. Unfortunately, the data is sparse early on (i.e., up to approximately Day 2600) and we cannot be certain of the proper behavior during that period, but the intensity is well-constrained for the rest of the observation period. We should also note that the data compiled in Jewell et al. (1981), realized with different facilities and instruments that sometimes probe different polarization states, do not focus on a single spectral feature but rather represent the integrated flux over a finite bandwidth. Despite these facts and the simplicity of our model, the oscillatory behavior of the intensity is relatively well-captured by the superradiance model. More precisely, the intensities of the last four maxima (at times beyond Day 2500) are reasonably well-matched by the curve, both in their relative intensities and times of occurrence.
We note that the superradiant system stemming from our calculations yields a cylindrical length $L=3.4 \times 10^{4} \mathrm{~cm}$, which is orders of magnitude shorter than corresponding scales expected for masers. It follows that, within the context of our model, a large number of superradiant large samples must be responsible for creating a radiation intensity strong enough to be detected during the flaring period (see Section 4.3 below). Also, the dephasing time $T^{\prime}$ needed to reproduce the data corresponds to approximately 7 years $\left(\sim 10^{8} \mathrm{~s}\right.$ and $\left.\simeq 10 \tau_{\mathrm{D}}\right)$ and points to conditions significantly less constraining than those previously calculated for a thermally relaxed gas, as expected. This implies the existence of significant velocity coherence over the length $L$ of a superradiant sample. This may not be surprising considering the Sobolev length for this source, which we evaluate to be $L_{\text {Sobolev }} \sim 10^{16} \mathrm{~cm}$ using previously published data on the velocity gradient found in U Ori's CSE (Nguyen-Q-Rieu et al. 1979). The relative smallness of a superradiant sample (i.e., $L / L_{\text {Sobolev }} \sim 10^{-12}$ ) is an indicator of small frequency shifts and longer dephasing timescales in the superradiant samples.

\subsection{The IRAS 18276-1431 Preplanetary Nebula}

IRAS 18276-1431 (OH17.7-2.0) is a preplanetary nebula with a detached CSE and a central star of a spectral type earlier than K5, long known for its strong $1612 \mathrm{MHz} \mathrm{OH}$ maser emission (Bowers 1974). Wolak et al. (2014) recently published the results of a monitoring campaign performed between 2002 and 2009, where emission from $\mathrm{OH}$ masers at 1612,1665 , and $1667 \mathrm{MHz}$ was measured twice monthly using the Nançay Radio Telescope. While a monotonic decay and rise in intensity were detected at $1665 \mathrm{MHz}$ and $1667 \mathrm{MHz}$, respectively, the integrated flux from the redshifted part of the $1612 \mathrm{MHz}$ spectrum revealed a significant intensity flare lasting approximately 6 years. Furthermore, during that same period 


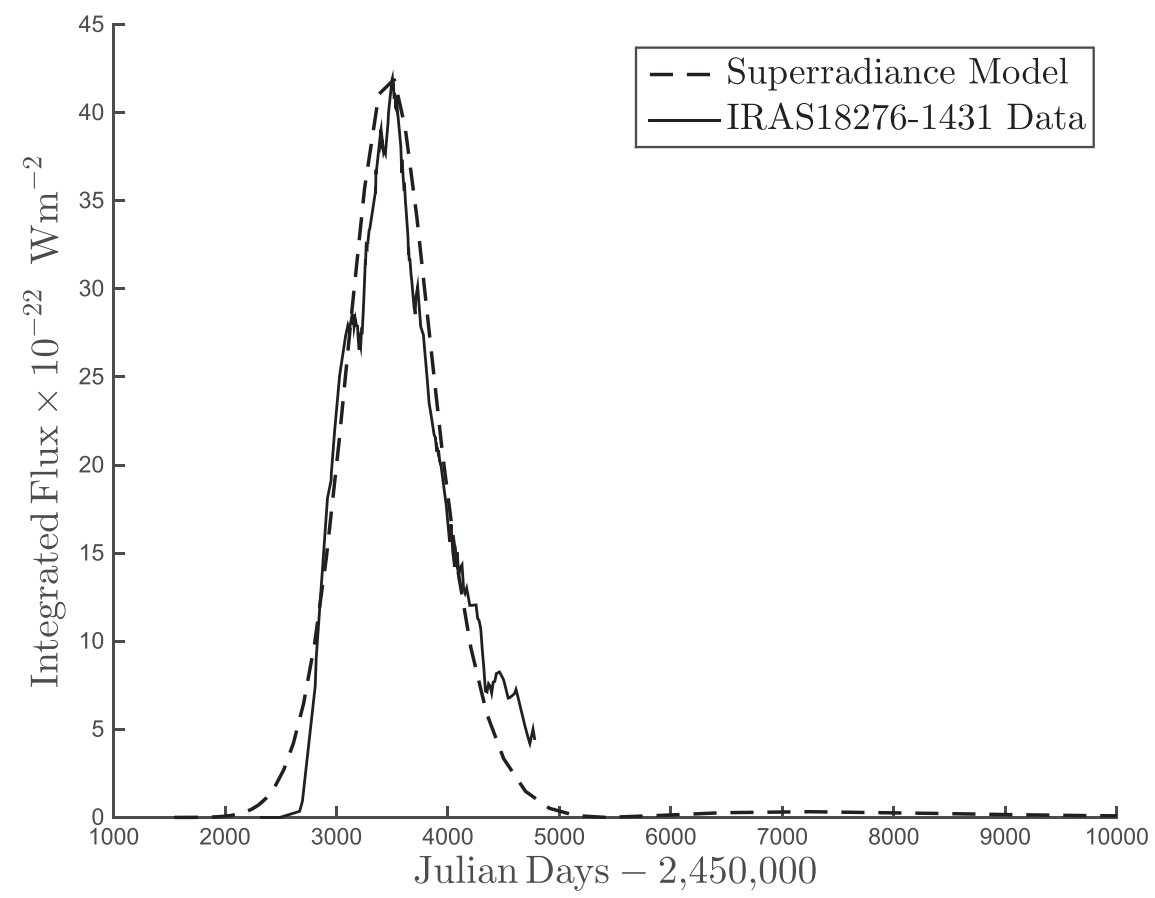

Figure 4. Superradiance intensity model (broken curve) with $T_{\mathrm{R}}=42$ days, $T^{\prime}=61 T_{\mathrm{R}}$, and $\theta_{0}=2.8 \times 10^{-4}$ rad, superposed on data from Wolak et al. (2014; solid curve) that was obtained during the 2002-2009 OH $1612 \mathrm{MHz}$ maser flaring episode of IRAS 18276-1431.

the blueshifted part of the spectrum only displayed a monotonic decay.

We show in Figure 4 the results of calculations using our superradiance model (broken curve) superposed to the data from Wolak et al. (2014; solid curve) for the aforementioned flaring episode of the $\mathrm{OH} 1612 \mathrm{MHz}$ line. Although the duration of the data does not allow us to determine if the burst of radiation taking place between approximately Day 2800 and Day 5000 is a single occurrence or part of a series of bursts, the longer duration of the flare ( 2000 days) in comparison to the case of U Ori and the expected limitation on the dephasing time $T^{\prime}$ seem to imply a single flare. Accordingly, our superradiance model yields $T_{\mathrm{R}}=42$ days, $\quad T^{\prime}=61 T_{\mathrm{R}}, \quad$ and $\theta_{0}=2 / \sqrt{N}=2.8 \times 10^{-4} \mathrm{rad}\left(\tau_{\mathrm{D}} \simeq 25 T_{\mathrm{R}}\right)$, and provides $\mathrm{a}$ rewardingly nice match to the data.

As for U Ori, we note that the superradiance cylinder length $L=5.2 \times 10^{3} \mathrm{~cm}$ is orders of magnitude shorter than those expected for masers, implying that large numbers of superradiant large samples are behind the measured intensity variation, while the required dephasing time $T^{\prime} \simeq 7$ years $\left(\sim 10^{8} \mathrm{~s}\right.$ and $\left.\simeq 2.4 \tau_{\mathrm{D}}\right)$ is markedly longer than the mean collision times previously calculated for $10^{4} \mathrm{~cm}^{-3}$ $\leqslant n_{\mathrm{H}_{2}} \leqslant 10^{6} \mathrm{~cm}^{-3}$ at $T=100 \mathrm{~K}$, as expected. And, once again, because of the small size of a superradiant sample $\left(L=5.2 \times 10^{3} \mathrm{~cm}\right)$ relative to typical values for the Sobolev length in the CSE of evolved stars, less constraining dephasing effects are anticipated.

\subsection{Transition between Maser and Superradiance Modes}

The data sets from Jewell et al. (1981) and Wolak et al. (2014) both indicate transitions between periods of (quasi) steady-state maser radiation and flaring episodes. Here we propose a scenario where such transitions could take place within the context of the superradiance model.
We know from our discussion in Section 2 that superradiance requires a dephasing time such that $T^{\prime}>\tau_{\mathrm{D}}$. We therefore surmise, because of the relationship between $\tau_{\mathrm{D}}$ and $T_{\mathrm{R}}$ given in Equation (17), the existence of a critical value $T_{\mathrm{R}, \text { crit }}$ for the characteristic timescale of superradiance, which cannot be exceeded for superradiance to take place. That is, super-

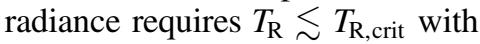

$$
T_{\mathrm{R}, \text { crit }}=\frac{4 T^{\prime}}{\left|\ln \left(\frac{\theta_{0}}{2 \pi}\right)\right|^{2}},
$$

which in turn can be manipulated to yield, using Equation (12), a corresponding critical value for the product of the inverted population density $n$ and the large sample length $L$. In other words, superradiance also implies a column density of inverted molecules $n L \gtrsim(n L)_{\text {crit }}$ with

$$
(n L)_{\text {crit }}=\frac{2 \pi}{3 \lambda^{2}} \frac{\tau_{\mathrm{sp}}}{T^{\prime}}\left|\ln \left(\frac{\theta_{0}}{2 \pi}\right)\right|^{2} .
$$

In the AGB phase, the mass-loss rate can change significantly during thermal pulsation periods, when the evolved star blows away its mass in the form of super winds, or when the CSE starts detaching from the star. These variations happen over relatively short timescales (i.e., on the order of a few years) and can result in correspondingly important changes in $L$ and $n$ in an $\mathrm{OH}$ large sample. However, variation in $n L$ does not necessarily require abrupt disruptions in the CSE and could happen more gradually over time or even because of local variations on smaller spatial scales.

Whatever the case may be, we can therefore imagine a situation where a region harboring an $\mathrm{OH}$ maser could experience a change in $n L$ that would push it above the critical value given in Equation (22). At that point, the region over which $n L>(n L)_{\text {crit }}$ would erupt into a superradiance mode that would overtake the maser process, since superradiance is a lot 
more efficient at radiating the energy stored in the sample (i.e., the superradiance radiation intensity scales with $N^{2}$ ). The initial maser radiation field itself could serve as a trigger for the superradiant event. Interestingly, our superradiance models for U Ori and IRAS $18276-1431$ show that $n L$ (or $T_{\mathrm{R}}$ and $\tau_{\mathrm{D}}$ ) is only a factor of a few (at most an order of magnitude for U Ori) higher than the critical value.

The timescales of the flaring events for U Ori and IRAS 18276-1431 indicate, however, that the whole maser region cannot at once act as a single coherent radiating system. Presumably, some other factor does not allow this to take place. For example, this could be because the level of velocity coherence is not high enough over the whole masing region to ensure that the corresponding dephasing time is sufficiently long to allow the entire region to act as a single superradiance system. On the other hand, velocity coherence is likely to be sufficient locally (i.e., on scales on the order of $10^{3}-10^{4} \mathrm{~cm}$ for the examples considered here) to allow for the corresponding region within the maser to break-up into a large number of smaller superradiance large samples. Our calculations presented in Figures 3 and 4, and their level of agreement with the corresponding data, provide credible evidence for such a scenario.

It is interesting to note that from an observational standpoint, a single superradiant volume over which $n L>(n L)_{\text {crit }}$ is unlikely to be resolvable in view of its spot size on the sky. This would be unusual for masers, which are often resolvable through high-resolution interferometry observations. This could also imply, based on the model discussed here, that an unresolvable source in a masing region exhibiting characteristics associated with superradiance (e.g., the ringing effect in the intensity variations) could be associated with a system composed of a single or a few superradiant sources operating within that region. However, as is implied by the discussion above, the converse is not necessarily true. That is, the fact that a source is spatially resolved does not imply that it cannot consist of a group composed of a large number of superradiant systems.

Finally, within the context of the model presented here, since a given region can make transitions between maser and superradiance modes we would expect similar proper motion properties to apply to both types of sources.

\section{CONCLUSION}

We have applied the concept of superradiance that was introduced by Dicke (1954) to the $\mathrm{OH}$ molecule $1612 \mathrm{MHz}$ spectral line that is often used for the detection of masers in the CSEs of evolved stars. Because the detection of $1612 \mathrm{MHz} \mathrm{OH}$ masers in the outer shells of envelopes of these stars implies the existence of a population inversion and a high level of velocity coherence, and that these are two necessary requirements for superradiance, we investigated whether or not superradiance can also take place in these regions. Our analysis suggests that superradiance provides a valid explanation for previous observations of intensity flares detected in that spectral line for the U Orionis Mira star (Jewell et al. 1981) and the IRAS 18276-1431 preplanetary nebula (Wolak et al. 2014). The confirmation of superradiance in these sources would not only reveal a new range of unexplored physical conditions in the
ISM, but on a more fundamental level also reveal the existence of coherent quantum mechanical systems and corresponding entangled states over length scales reaching a few times $10^{4} \mathrm{~cm}$.

We are grateful to P. Wolak for making his data on IRAS 18276-1431 available to us. M.H.'s research is funded through the NSERC Discovery Grant and the Canada Research Chair programs.

\section{REFERENCES}

Baan, W. A., Wood, P. A. D., \& Haschick, A. D. 1982, ApJL, 260, L49 Benedict, M. G., Ermolaev, A. M., Malyshev, V. A., Sokolov, I. V., \& Trifonov, E. D. 1996, Super-radiance Multiatomic Coherent Emission (Bristol: IOP Publishing Ltd)

Bertolotti, M. 2015, Masers and Lasers: an Historical Approach (Boca Raton, FL: CRC Press)

Bowers, P. F. 1974, A\&AS, 31, 127

Cimerman, M. 1979, ApJL, 228, L79

Darling, J., \& Giovanelli, R. 2002, ApJ, 124, 100

Dicke, R. H. 1954, PhRv, 93, 99

Draine, B. T. 2011, Physics of the Interstellar and Intergalactic Medium (Princeton, NJ: Princeton Univ. Press)

Elitzur, M. 1992, Astronomical Masers (Dordrecht: Kluwer)

Elitzur, M., Goldreich, P., \& Scoville, N. 1976, ApJ, 205, 384

Fix, J. D. 1979, ApJL, 232, L39

Goldreich, P., \& Scoville, N. 1976, ApJ, 205, 144

Gray, M. 1999, RSPTA, 357, 3277

Gray, M. 2012, Maser Sources in Astrophysics (New York: Cambridge Univ. Press)

Gray, M. D., Howe, D. A., \& Lewis, B. M. 2005, MNRAS, 364, 783

Gross, M., \& Haroche, S. 1982, PhR, 95, 301

Harvey, P. M., Bechis, K. P., Wilson, W. J., \& Ball, J. A. 1974, ApJS, 27, 331

He, J. H. 2005, NewA, 10, 283

Irwin, J. A. 2007, Decoding the Cosmos (Chichester: Wiley)

Jewell, P. R., Webber, J. C., \& Snyder, L. E. 1981, ApJ, 249, 118

Jewell, P. R., Webber, J. C., Snyder, L. E., \& Elitzur, M. 1979, ApJS, 41, 191

Karttunen, H. 2007, Fundamental Astronomy (Berlin: Springer Science \& Business Media)

Kylafis, N. D., \& Norman, C. A. 1990, ApJ, 350, 209

Lamers, H. J., \& Cassinelli, J. P. 1999, Introduction to Stellar Winds (Cambridge: Cambridge Univ. Press)

Litvak, M. M. 1969, ApJ, 156, 471

Lockett, P., \& Elitzur, M. 2008, ApJ, 677, 985

McBride, J., Heiles, C., \& Elitzur, M. 2013, ApJ, 774, 1

McGee, R. X., Robinson, B. J., Gardner, F. F., \& Bolton, J. G. 1965, Natur, 208, 1193

Nguyen-Q-Rieu, Laury-Micoulaut, C., Winnberg, A., \& Schultz, G. V. 1979, A\&A, 75, 351

Offer, A. R., van Hemert, M. C., \& van Dishoeck, E. F. 1994, JChPh, 100,362

Pataki, L., \& Kolena, J. 1974, in Bulletin of the American Astronomical Society, A change in the nature of the $\mathrm{OH}$ emission from U Orionis, Vol. 6,340

Rajabi, F., \& Houde, M. 2016, ApJ, 826, 216 Paper I

Reid, M. J., Muhleman, D. O., Moran, J. M., Johnston, K. J., \& Schwartz, P. R. 1977, ApJ, 214, 60

Souers, P. C. 1986, Hydrogen Properties for Fusion Energy (London: Univ. California Press Ltd)

Stahler, S. W., \& Palla, F. 2008, The Formation of Stars (Weinheim: Wiley-VCH)

Turner, B. E. 1970, JRASC, 64, 221

Weaver, H., Williams, D. R., Dieter, N. H., \& Lum, W. T. 1965, Natur, 208, 29

Weinreb, S., Barrett, A. H., Meeks, M. L., \& Henry, J. C. 1963, Natur, 200, 4909

Wittke, J. P., \& Dicke, R. H. 1956, PhRv, 103, 620

Wolak, P., Szymczak, M., Bartkiewicz, A., \& Gérard, E. 2014, in Proc. 12th European VLBI Network Symp. and Users Meeting (EVN 2014), 116 